\title{
TP d'électrotechnique : onduleur triphasé 24 volts
}

\author{
Imbaud Joël, Vernier David, Nikolay Vorobyev \\ joel.imbaud@ens2m.fr \\ ENSMM, 26 Rue de l’Epitaphe, 25030 Besançon cedex, France
}

\begin{abstract}
RESUME : Cet article présente la mise en place d'une séance de travaux pratiques d'électrotechnique portant sur la mise en œuvre d'un onduleur triphasé 24 volts et de son moteur asynchrone triphasé associé. Cette manipulation est mise à disposition à des élèves de $2^{\text {ème }}$ année d'école d'ingénieur dans le parcours Electronique Automatique Optique (EAO). Le logiciel Pspice est utilisé pour simuler l'interface de commande de la manipulation. Les commentaires des élèves pour une année d'expériences sont analysés.
\end{abstract}

Mots clés : Travaux Pratiques, onduleur triphasé, moteur asynchrone, électronique de puissance, retour d'expérience.

\section{INTRODUCTION}

L'École Nationale Supérieure de Mécanique et des Microtechniques (ENSMM) est une école d'ingénieurs généralistes avec une dominante mécanique et microtechniques. Actuellement la deuxième année de l'ENSMM (Bac + 4) est constituée de deux parcours de 90 heures de formation spécifique (fig. 1): parcours Mécanique et Matériaux (MM) et parcours Electronique Automatique Optique (EAO). Ces deux parcours contiennent 5 modules dans lesquels les étudiants doivent en choisir 3. Le module EAO2 porte spécifiquement sur une initiation à l'électrotechnique aux travers de l'étude du réseau électrique, des machines tournantes et des onduleurs. Les enseignements, dans ce module de 30 heures se répartissent entre 10 heures de cours, 12 heures de travaux dirigés et 8 heures de travaux pratiques. Durant les séances de TP, 4 heures sont destinées à la manipulation présentée dans ce papier. Elle a pour but de caractériser un moteur triphasé $24 / 42$ volts de type asynchrone en le pilotant avec un onduleur simplifié.

\begin{tabular}{|c|c|c|c|c|c|c|}
\hline \multicolumn{7}{|c|}{$\begin{array}{c}\text { PARCOURS : Semestre VERT } \\
\text { 2 parcours au choix (3 cours parmi 5) } \\
\text { TOTAL: } 90 \mathrm{Ch}-6 \text { ECTS } \\
\text { (16 semaines : Automne : Septembre - Janvier ou Printemps : Fevrier - Juin) } \\
\text { (Disciplines, Volumes horaires, Responsable de thematique) }\end{array}$} \\
\hline & & Total & Cours & TD & TP & ECTS \\
\hline \multicolumn{7}{|c|}{$\begin{array}{l}\text { Parcours MM : Mécanique - Matériaux } \\
3 \text { cours à choisir parmi } 5 \text { ( } 90 \mathrm{~h}-6 \mathrm{ECTS})\end{array}$} \\
\hline MM1 & $\begin{array}{l}\text { Mécanique des Fluides } \\
\text { V. Guicheret } \\
\text { examen (3/4) + TP (1/4) }\end{array}$ & 30 & 10 & 8 & 12 & 2 \\
\hline MM2 & $\begin{array}{l}\text { Mécanique Vibratoire } \\
\text { S. Mallet (Automne) / M. Ouisse (Printemps) } \\
\text { 1 partiel }\end{array}$ & 30 & 8 & 10 & 12 & 2 \\
\hline MM3 & $\begin{array}{l}\text { Surfaces et Interfaces en science des matériaux } \\
\text { Jamal Takadoum } \\
\text { partiel (1): moyenne de } \mathbf{2} \text { notes }\end{array}$ & 30 & 18 & 12 & 0 & 2 \\
\hline MM4 & $\begin{array}{l}\text { Matériaux hautes performances et Biomatériaux } \\
\text { Guy Monteil } \\
\text { partiel (1): moyenne de } 3 \text { notes }\end{array}$ & 30 & 20 & 10 & 0 & 2 \\
\hline MMS & $\begin{array}{l}\text { Microtechniques } \\
\text { Stephane Gauthier } \\
\text { partiel }(2 / 3)+\text { compte rendu de TP (1/3) }\end{array}$ & 30 & 10 & 4 & 16 & 2 \\
\hline \multicolumn{7}{|c|}{$\begin{array}{l}\text { Parcours EAO : Electronique - Automatique - Optique } \\
3 \text { cours à choisir parmi } 5 \text { (90h-6 ECTS) }\end{array}$} \\
\hline EAO1 & 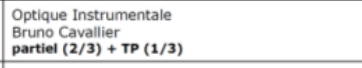 & 30 & 10 & 8 & 12 & 2 \\
\hline $\mathrm{EAO} 2$ & $\begin{array}{l}\text { Electrotechnique } \\
\text { Joel Imbaud } \\
\text { partiel }(\mathbf{3} / \mathbf{4})+\operatorname{TP}(\mathbf{1 / 4 )}\end{array}$ & 30 & 10 & 12 & 8 & 2 \\
\hline $\mathrm{EAO} 3$ & \begin{tabular}{|l} 
Electronique Numérique \\
Fabrice Sthal \\
partiel (2/5) + TD (2/5) + TP (1/5)
\end{tabular} & 30 & 10 & 12 & 8 & 2 \\
\hline EAO4 & $\begin{array}{l}\text { Programmation Orientée Objet } \\
\text { Gulliaume Laurent } \\
\text { examen }(\mathbf{1 / 2})+\text { mini-projet }(\mathbf{1 / 2})\end{array}$ & 30 & 8 & 0 & 22 & 2 \\
\hline EAOS & $\begin{array}{l}\text { Automatique Avancée } \\
\text { Yassine Haddab } \\
\text { examen }(\mathbf{2} / \mathbf{3})+\mathrm{TP}(\mathbf{1 / 3})\end{array}$ & 30 & 10 & 8 & 12 & 2 \\
\hline
\end{tabular}

fig 1 : Choix des parcours de $2^{\text {ème }}$ année.

\section{DESCRIPTION DU COURS}

Le cours décrit le fonctionnement général et les grandeurs du réseau électrique français, ainsi que le calcul des différentes puissances apparente, active et réactive en monophasé et triphasé pour les deux types de câblages étoile et triangle. Le principe de fonctionnement des redresseurs et des hacheurs séries/parallèles/en pont en H leur est présenté. Une initiation aux bases du magnétisme est ensuite proposée. Quelques rappels sur le calcul des forces, travails et moments d'inertie sont effectués dans le but de sensibiliser les étudiants à la récupération d'énergie. La suite se fait avec les machines tournantes, débutant par la présentation du principe des moteurs à inductions, l'étude particulière du moteur synchrone, du moteur asynchrone et enfin du moteur «brushless ». C'est aussi à cette occasion que le principe de l'onduleur triphasé pour la variation de vitesse est décrit. La présentation du moteur à courant continu et du moteur pas à pas fait suite. Le cours se conclu sur la présentation des risques électriques et des habilitations associées à leurs obligations légales.

\section{FONCTIONNEMENT}

Le principe de l'onduleur triphasé est extrêmement simple, pas de commande complexe, il est uniquement basé sur la création de créneaux carrés. Le système triphasé est généré à l'aide d'un automate synchrone à 6 états constituée de trois bascules D (fig. 2).

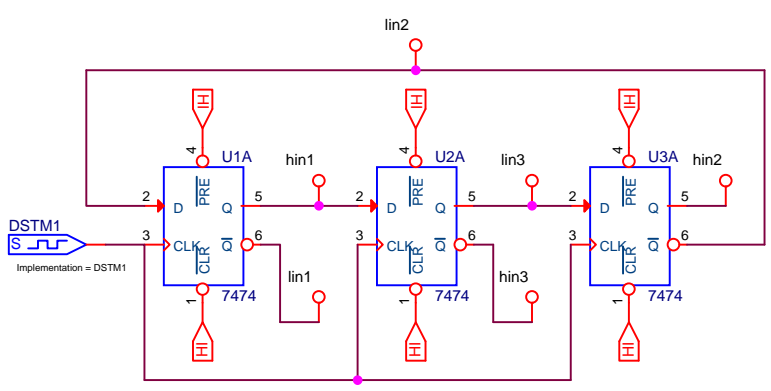

fig 2 : Schéma de principe de la machine synchrone générant la commande des transistors du pont pour la génération des signaux triphasés. 
Cet ensemble de bascules commandent un étage de puissance constitué de six transistors IGBT. Le schéma de principe est rappelé en fig. 3. Ce schéma est celui utilisé pour la simulation fonctionnelle de l'ensemble sous Pspice.

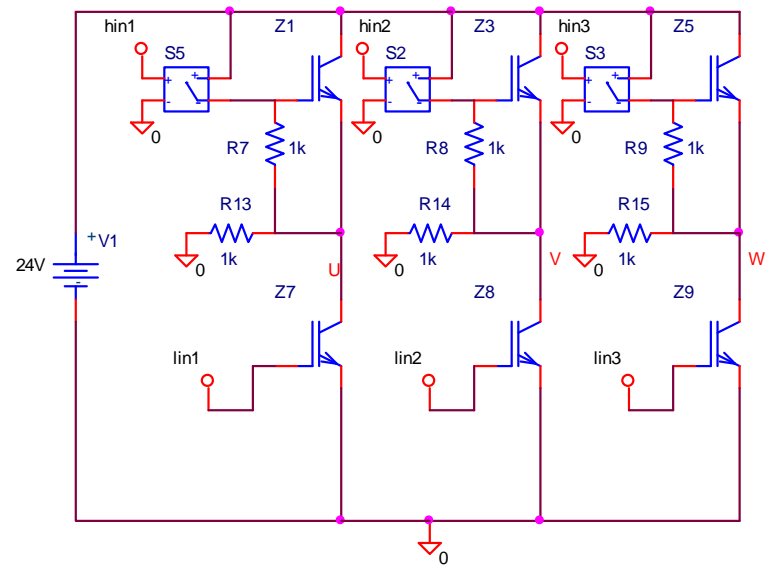

fig 3 : Schéma de principe de l'étage de puissance pour la simulation fonctionnelle.

Les tensions générées en $\mathrm{U}, \mathrm{V}$ et $\mathrm{W}$ correspondent à un système de tension triphasé. La fréquence résultante est celle de l'horloge des bascules D divisée par 6. Cette diminution de fréquence est due au fait que l'automate synchrone comporte 6 états. Les tensions composées sont illustrées en fig. 4.

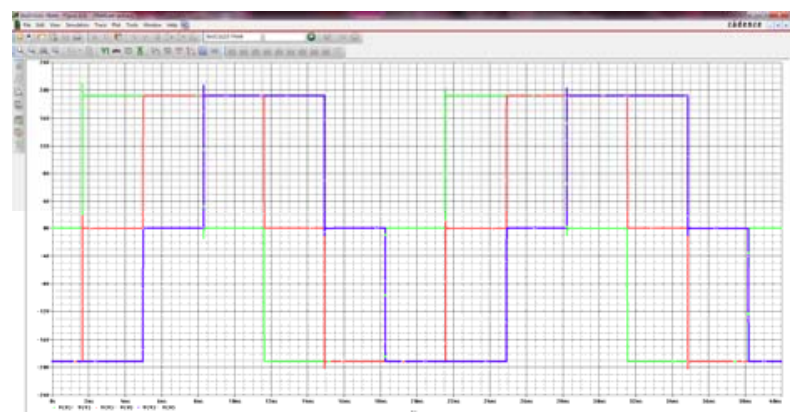

fig 4 : Tensions composées $U-V, U-W$ et $V-W$ du système triphasé.

En faisant varier la valeur de l'alimentation de puissance, il est possible de contrôler la valeur efficace des tensions générées. Il en est de même avec la fréquence, l’horloge de référence étant fournie par un générateur.

Cet onduleur est ensuite connecté à un moteur asynchrone triphasé $24 \mathrm{~V} / 42 \mathrm{~V}$ (fig. 5) produit par la société Langlois [1]. Ce moteur fait partie d'un banc de test comprenant un volant d'inertie de $0,011 \mathrm{~kg} \cdot \mathrm{m}^{2}$ et d'un frein à poudre accompagné d'une balance et d'une dynamo tachymétrique (fig. 6).

\section{MOTEUR ASYNCHRONE TRI A CAGE}

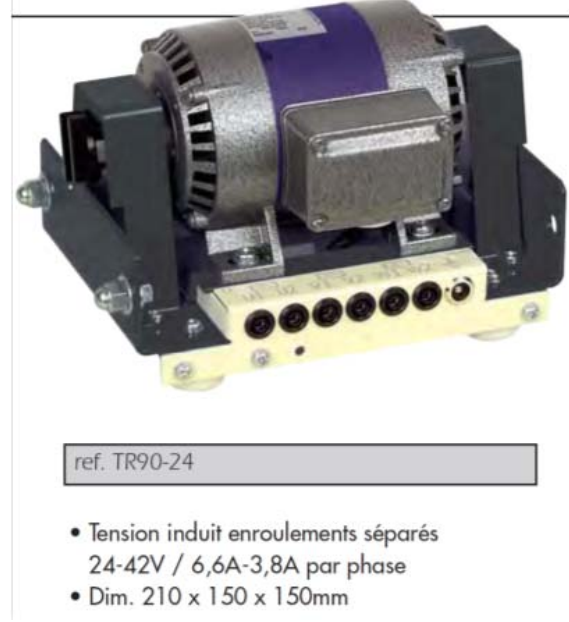

fig 5 : Moteur asynchrone du catalogue.

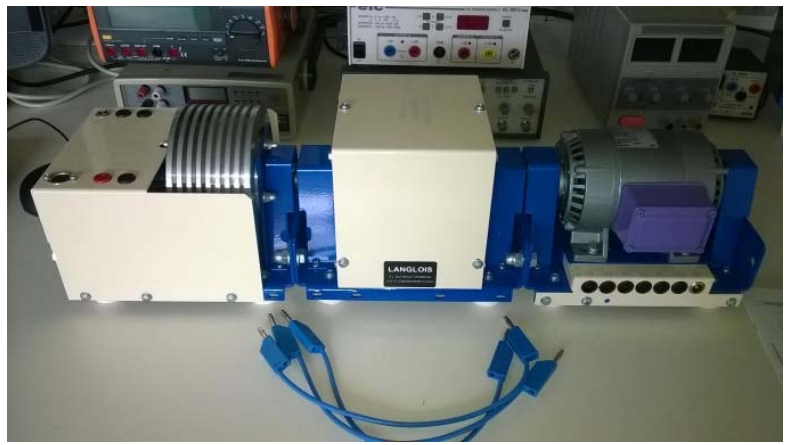

fig 6 : Constitution du banc moteur asynchrone.

La partie puissance est alimentée par une alimentation variable $0-30 \mathrm{~V} / 6$ A max et la partie commande par une alimentation fixe séparée.

\subsubsection{Objectif}

L'objectif du TP est de faire manipuler aux étudiants l'ensemble onduleur + banc moteur dans le but de mieux appréhender le comportement du moteur asynchrone.

La première partie du TP porte sur la détermination des différents éléments du modèle linéarisé ramené au stator de la machine (fig. 7). Ce modèle fait apparaître la résistance du stator $R_{1}$, la bobine à noyau de fer $L_{1}$ et $\mathrm{R}_{\mathrm{F}}$, l'inductance de fuite $\mathrm{l}_{2}$ et la résistance fictive représentant la puissance transmise $R_{2} / g$ avec $R_{2}$ la résistance du rotor ramenée au stator.

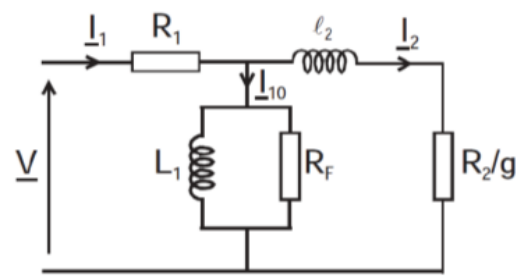

fig 7 : Modèle linéarisé ramené au stator pour une phase. 
Les étudiants comparent leurs mesures avec les données et les tests du constructeur (fig. 8). Ensuite, ils calculent à partir de l'essai à vide et des puissances active et réactive la partie bobine à noyau de fer du modèle. Ils effectuent au même moment la mesure de la résistance du stator.

\begin{tabular}{|c|c|c|c|c|}
\hline \multicolumn{5}{|c|}{$\begin{array}{l}\text { MOTEUR TRIPHASE à CAGE } \\
\text { TR90/24 }\end{array}$} \\
\hline \multicolumn{5}{|c|}{ CARACTERISTIQUES PRINCIPALES : } \\
\hline \multirow{4}{*}{\multicolumn{3}{|c|}{$\begin{array}{l}\text { Résistance entre phase à froid : } \\
\text { Résistance entre phase à chaud : } \\
\text { Intensité nominale d'utilisation en Etoile : } \\
\qquad \text { en Triangle : }\end{array}$}} & $0.93 \Omega$ & \\
\hline & & & $1.02 \Omega$ & \\
\hline & & & $3.8 \mathrm{~A}$ & \\
\hline & & & & \\
\hline \multirow{2}{*}{\multicolumn{5}{|c|}{$\begin{array}{l}\text { Vitesse nominale à vide, sans aucune machine accouplée : } 2990 \mathrm{tr} / \mathrm{min} \text { sous } 50 \mathrm{~Hz} \\
\text { Intensité à vide : } 2.32 \mathrm{~A} \text { (Couplage Etoile } 42 \mathrm{~V} \text { entre phases) } \\
\text { Tableau réalisé avec les constantes suivantes } \\
\text { - tension secteur fixe de } 42 \text { volts entre phases et } 50 \mathrm{~Hz} \\
\text { - couplage Etoile }\end{array}$}} \\
\hline & & & & \\
\hline \multicolumn{5}{|c|}{ Le rendement est calculé avec le moteur qui entraine une génératrice shunt de même puissance } \\
\hline \multicolumn{5}{|c|}{ TABLEAU DE CARACTERISTIQUES en CHARGE } \\
\hline Charge & $\begin{array}{c}\text { Intensite par phase } \\
\text { en A }\end{array}$ & $\begin{array}{c}\text { Vitesse } \\
\text { en tr/min }\end{array}$ & $\begin{array}{l}\text { Rendement en \% } \\
\quad \text { de PuPf }\end{array}$ & Cosinus $\varphi$ \\
\hline $\begin{array}{c}\text { premier point } \\
\text { de mesure à vide }\end{array}$ & 2.32 & 2990 & 1 & 0.36 \\
\hline $\begin{array}{l}\text { deuxième point } \\
\text { de mesure }\end{array}$ & 2.48 & 2942 & 17 & 0.57 \\
\hline $\begin{array}{c}\text { troisième point } \\
\text { de mesure }\end{array}$ & 2.62 & 2919 & 28. & 0.63 \\
\hline $\begin{array}{l}\text { quatrieme point } \\
\text { de mesure }\end{array}$ & 2.86 & 2866 & 40 & 0.72 \\
\hline $\begin{array}{l}\text { cinquième point } \\
\text { de mesure }\end{array}$ & 2.94 & 2853 & 38 & 0.77 \\
\hline $\begin{array}{l}\text { sixième point } \\
\text { de mesure }\end{array}$ & 3.05 & 2841 & 38 & 0.78 \\
\hline $\begin{array}{l}\text { septième point } \\
\text { de mesure }\end{array}$ & 3.2 & 2814 & 38 & 0.80 \\
\hline $\begin{array}{l}\text { huitième point } \\
\text { de mesure }\end{array}$ & 3.4 & 2753 & 36 & 0.83 \\
\hline $\begin{array}{l}\text { neuvieme point } \\
\text { de mesure }\end{array}$ & 3.6 & 2710 & 33 & 0.83 \\
\hline
\end{tabular}

fig 8 : Données constructeur du moteur triphasé.

La mesure de la partie rotor ramenée au stator est effectuée rotor bloqué (par le frein à poudre) sous une alimentation réduite. Le courant dans une phase doit être ajusté pour être équivalent au courant en fonctionnement nominal. Il est mesuré en intercalant une résistance de shunt entre une sortie de l'onduleur et une phase du moteur. Avec le déphasage entre tension et courant dans une phase, il est alors possible de déterminer les puissances actives et réactives, les étudiants peuvent alors calculer l'inductance de fuite et la résistance fictive.

Une fois le travail de modélisation effectué, les étudiants doivent lancer une campagne de mesure à différentes fréquences de travail en essayant de garder au mieux le rapport tension efficace sur fréquence constant (dans la limite de plage de l'alimentation). Ils doivent constater que la zone de fonctionnement du moteur est translatée par rapport à elle-même et qu'il est ainsi possible de régler la vitesse. Le couple résis- tant est appliqué au moteur par l'intermédiaire du frein à poudre. L'étudiant remonte au couple appliqué au moteur par la mesure du glissement $g$ et de la relation suivante :

$$
T_{E M}=\frac{3 p V_{E f f}^{2}}{\omega} \frac{R_{2}}{\frac{R_{2}^{2}}{g}+g\left(l_{2} \omega\right)^{2}}
$$

Les étudiants refont les mesures pour chaque type de couplage étoile et triangle comme indiqué sur le manuel constructeur fig. 9.

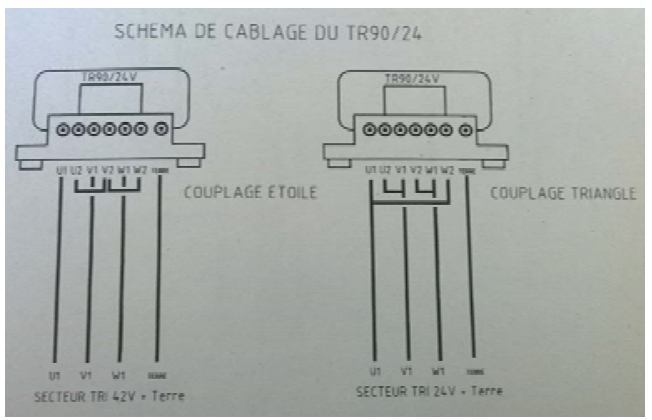

fig 9 : Schéma de câblage Etoile/triangle de la machine.

Les valeurs calculées à partir de l'expérimentale sont listées dans le tableau suivant :

\begin{tabular}{|c|c|c|}
\hline Eléments & Valeurs & Unités \\
\hline $\mathrm{R}_{1}$ & 1 & Ohm \\
\hline $\mathrm{R}_{\mathrm{F}}$ & 28,7 & Ohms \\
\hline $\mathrm{L}_{1}$ & $35 \mathrm{~m}$ & Henrys \\
\hline $\mathrm{R}_{2}$ & 1,2 & Ohms \\
\hline $\mathrm{l}_{2}$ & $60 \mathrm{~m}$ & Henrys \\
\hline
\end{tabular}

\section{MISE EN OEUVRE}

Les TP sont effectués sur des paillasses équipées de façon uniforme. Elles sont composées d'un ordinateur relié au réseau avec accès à tous les logiciels du parc informatique, de deux multimètres de table, d'un oscilloscope deux voies, d'un GBF, d'une alimentation ajustable faible puissance et d'une alimentation variable de puissance (fig. 10).

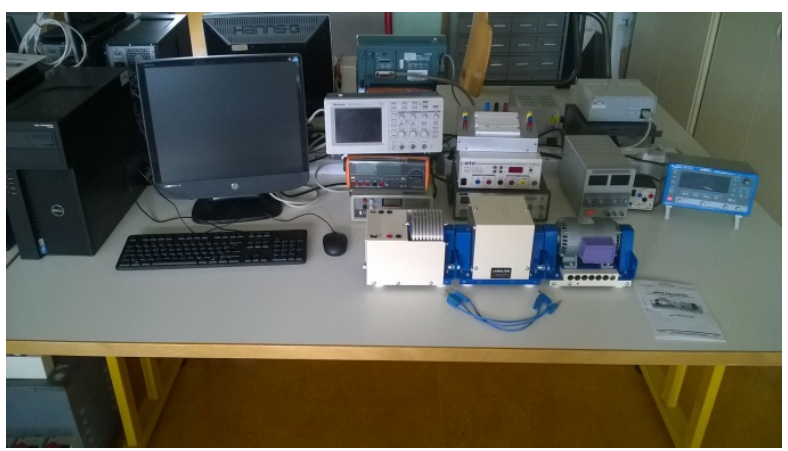

fig 10 : Paillasse de TP avec son équipement. 
Les paillasses équipées de ce banc moteur sont au nombre de 4, il donc été décider de réaliser 5 onduleurs pour en avoir un de rechange en cas de casse.

La conception des cartes d'onduleur a été faite de façon à ce que la tenue mécanique de la carte soit la plus robuste possible. Elle est équipée d'un radiateur largement dimensionné pour évacuer la chaleur des six transistors du pont (fig. 11).

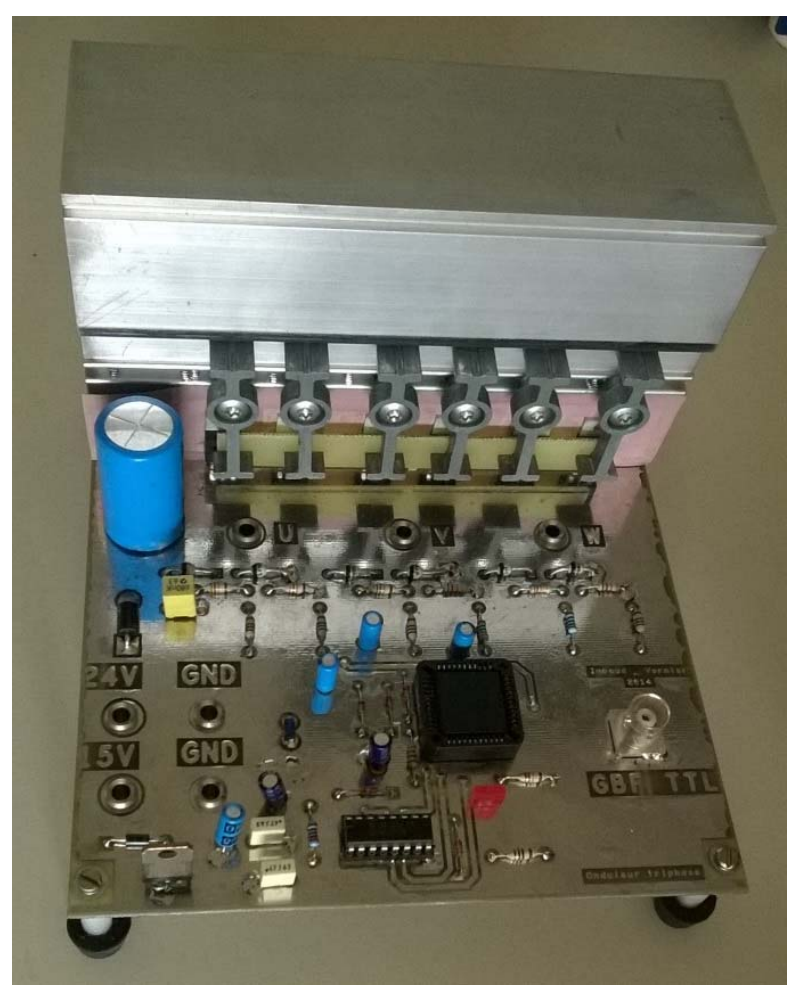

fig 11 : Carte de l'onduleur triphasé.

Le schéma de cette carte est basé sur le composant IRS2332 de chez International Rectifier [2] et d'une note d'application du même fabricant [3], dont le schéma est donné fig. 12.

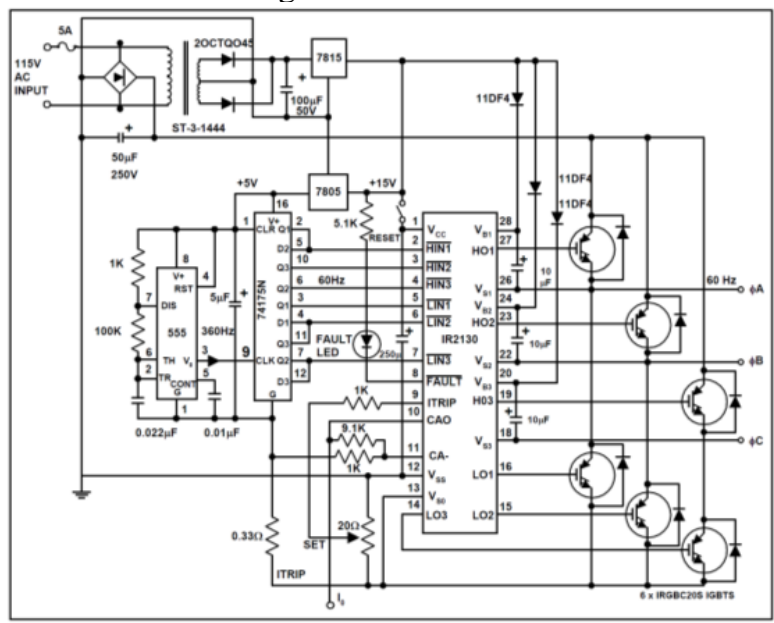

fig 12 : Schéma de base de la note d'application d'International Rectifier.
Le schéma a été simplifié en supprimant la partie mesure de courant en pied de pont et en externalisant l'horloge. Les alimentations du pont et de la partie commande ont été séparées. Des diodes de protections d'inversion de polarités et de surtension sur l'entrée GBF sont présentes. Les schémas et le dessin de la carte ont été réalisés sous Orcad [4]. Le schéma complet est donné fig. 13.
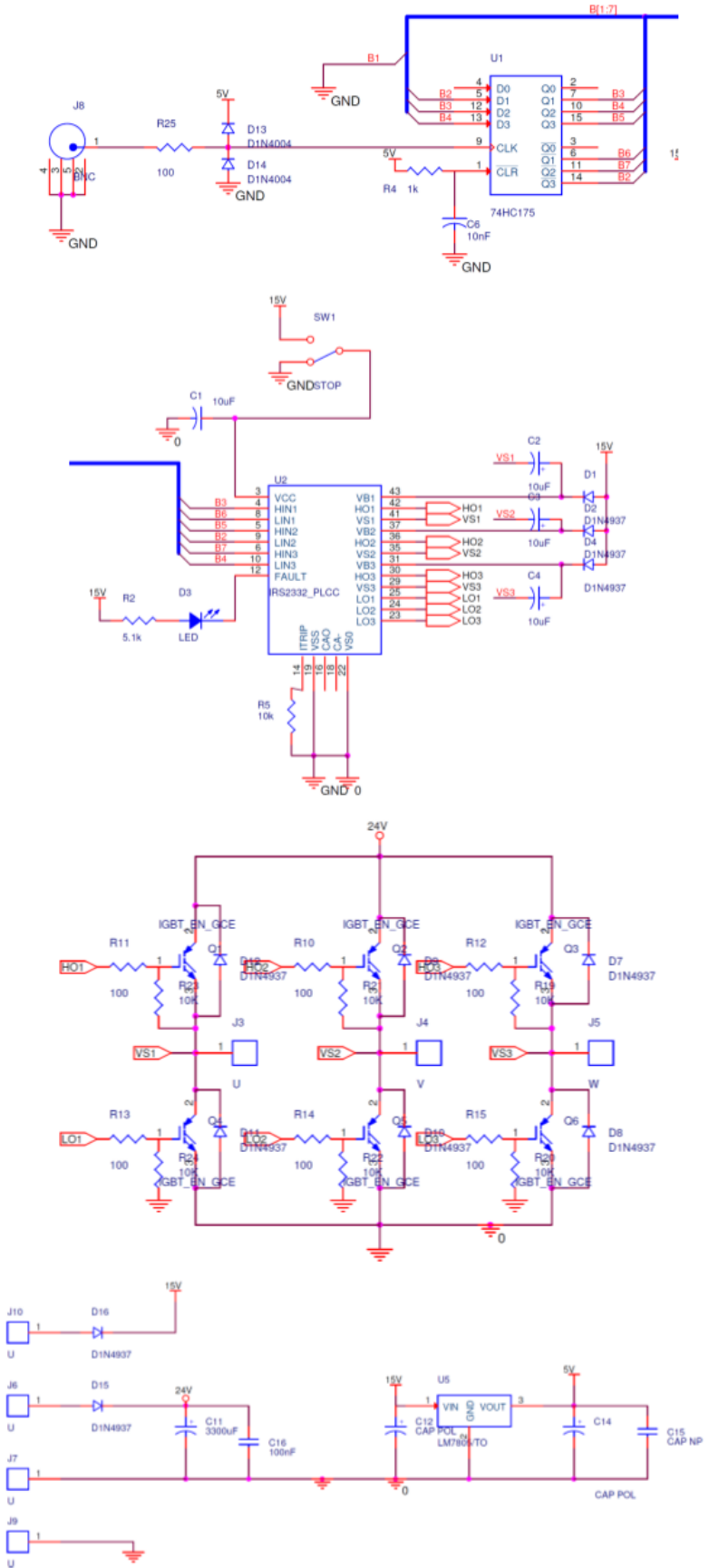

fig 13 : Schéma complet de l'ensemble de l'onduleur.

\section{ASPECT PEDAGOGIQUE}

Environ 15 élèves ont effectué le TP sur cette année, ils ont eu lieu début juin. 


\subsection{Point de vue des élèves}

A la fin de chaque TP, il a été engagé un échange sous la forme d'une discussion afin de commenter ce nouveau TP. Les étudiants sont globalement très satisfaits. Dans les commentaires, les points positifs sont :

- pouvoir relier une simulation d'électronique avec un objet réel simultanément

- $\quad$ voir un système dont le fonctionnement est à la portée de leurs analyses.

Ils peuvent mieux appréhender le contrôle moteur et ce TP est le parfait tremplin pour aller vers des commandes plus complexes (PWM, transformée de Park...) et les relier au monde de l'automatisme.

\subsection{Point de vue de l'enseignant}

Les courants qui circulent dans la carte électronique sont relativement importants. Au démarrage du TP, il est donc nécessaire de bien expliquer les branchements à réaliser entre les parties commande et puissance. Il faut savoir que le driver (IRS2332) est l'élément le plus sensible de la carte. Au cours des séances de TP, 2 ont été grillés. Pour pouvoir les remplacer facilement, ils ont été insérés dans des supports de type PLCC. Une autre cause de problème a été attribuée à des diodes de roue libre insuffisamment dimensionnées.

Ce TP était la toute première version. Par la suite, les problèmes précédemment évoqués ont été corrigés sur l’ensemble des cartes réalisées.

\section{CONCLUSION}

Ce TP est un parfait exemple d'onduleur triphasé très simple. Il permet aux étudiants de découvrir l'électronique de puissance et d'entrevoir ses applications.

Le TP permet aussi de mieux comprendre le fonctionnement du moteur asynchrone triphasé et son comportement en fonction de la fréquence du réseau.

Enfin, le point fort de ce TP est qu'il permet aux étudiants de découvrir un système d'onduleur « ouvert » et accessible pour un débutant alors que les systèmes du commerce sont très " boîte noire » et facilement compréhensible.

\section{Bibliographie}

[1] http://www.langlois-france.com, site internet de Langlois.

[2] http://www.irf.com, site internet du fabricant International Rectifier.

[3] Application Note AN-985, "Six-Output 600V MGDs

Simplify 3-Phase Motor Drives", International Rectifier.

[4] http://www.orcad.com, site internet de l'éditeur du logiciel Orcad. 